East African Medical Journal Vol. 85 No. 9 September 2008

PATIENT TRANSFER PRACTICES BY HOSPITALS IN WESTERN KENYA

R. T. Kuremu, MBChB, MMed (Surg), MMed Sc. (Paed Surg), FCS (ECSA) Senior Lecturer, Department of Surgery and Anaesthesiology, C. N. Tenge, MBChB, MMed (Paed.), Lecturer, Department of Child Health and Paediatrics, Moi University, School of Medicine, P. O. Box 4606-30100, Eldoret, Kenya, G. G. Wakuloba, BDS, MPH, Senior Dental Officer and A. Wambati, Dip. (Nursing), Adv. Dip (Nursing), Cert. (Trauma and Emergency Care), Moi Teaching and Referral Hospital, P. O. Box 3 - 30100, Eldoret, Kenya

Request for reprints to: Dr. R. T. Kuremu, Department of Surgery and Anaesthesiology, Moi University, School of Medicine, P. O. Box 4606 - 30100, Eldoret, Kenya

\title{
PATIENT TRANSFER PRACTICES BY HOSPITALS IN WESTERN KENYA
}

\author{
R. T. KUREMU, C. N. TENGE, G. G. WAKULOBA and A. WAMBATI
}

\begin{abstract}
Background: Patients who are critically ill and those requiring emergency care are transported within and between hospitals on a regular basis seeking diagnostic or therapeutic services not available at the bed side or within the referring institution. The emergency of specialty systems often determines the ultimate destination of patients rather than proximity of facility and this has heightened the need for patient transfer. To achieve a favorable outcome, it is necessary to ensure that any transfer is carried out safely and effectively with minimum disruption of the continuum of care.

Objectives: To determine the gap between existing knowledge of patient transfer principles and the practice by hospitals in Western Kenya referring patients to Moi Teaching and Referral Hospital (MTRH).

Design: Cross-sectional descriptive study.

Setting: Accident and emergency department at MTRH.

Subjects: Patients transferred in over a period of six months for critical/emergency care.

Results: Evaluation was done for 97 transfers during the six months period. Age ranged from four days old to 70 years with a median of 28 years. A wide spectrum of diseases were seen. However in order of frequency the leading five were; trauma and accidents, vascular disorders, infections; anaemia and malignancies. Of the infections, respiratory infections topped the list with pulmonary tuberculosis as the leading disease entity. Majority of patients $43(44 \%)$ were referred within 24 hours of being seen at the primary hospital. Only $56 \%$ were transported by ambulance; appropriate escort(nurse) was provided in $60 \%$; documentation was provided in $85 \%$; monitoring enroute was done in $24 \%$; warmth was provided in $62 \%, 27 \%$ were dehydrated requiring resuscitation; respiratory support was inadequate as only $14 \%$ (of those who required) had airway and $32 \%$ had oxygen provided; intravenous fluids were provided in $34 \%$ of those who required; nasogastric intubation was provided in $30 \%$ of those who required; urethral catheterisation was provided in $23 \%$ of those who required; $50 \%$ of those with long bone fractures were splinted and only $3 \%$ of those who required cervical spine stabilisation had cervical collar.

Conclusion: There was significant failure by hospitals in Western Kenya in the application of principles of patient transfer while referring patients to MTRH.
\end{abstract}

\section{INTRODUCTION}

Process or patient transfer should not introduce a hiatus in therapeutic and monitoring continuum (l) if the patient is to eventually benefit from the decision of transfer. Few hospitals have the capacity to provide comprehensive range of medical services (2), while in this country specialised medical services are centralised hence is mandatory that patients who require them are transferred to where they are available. 
As observed elsewhere in the developing world, transfer of patients provides a set of problems different from the developed world. Hospitals are far away, poorly staffed and inadequately equipped (3). Communication network is also poor. The process is hence fraught with many pitfalls when moving critically ill patients. The process of transfer is complex, difficult and may contribute to increased morbidity, mortality and may delay definitive treatment $(4,5)$.

To achieve the desired quality of care all concerned have to efficiently play their roles. When principles are applied, complications associated with transportation of the critically ill and emergency transfers are minimised and ensure optimal benefit to the patient. It saves time and resources, and effort will be channeled to definitive diagnostic / therapeutic procedures other than doing the routine care and resuscitation of patients referred in. The ultimate goal is to match patient needs with appropriate knowledge, skills, equipment, and infrastructure to enable safe, effective and efficient transfer.

Anecdotal evidence suggested that there was a gap in existing knowledge and the practice in the application and adherence to principles of patient transfer process in the catchments area of MTRH. This study was therefore undertaken to evaluate the process of patient transfer and determine factors affecting the achievement of desired practice. No similar study has been done in this region.

\section{MATERIALS AND METHODS}

This cross-sectional descriptive study was carried out at the accident and emergency department of MTRH. Patients transferred in for critical or emergency care were included. Consent was sought from patients or guardians as was appropriate. A questionnaire was filled detailing specific procedures that needed to have been done by referring facility. The observation made was whether this had been done or not as the patient arrived in the accident/ emergency unit. The escorts were recruited to complete the questionnaire on reasons for failure to apply the necessary measures. Immediate feedback was given to the escorts / health personnel concerning the value of the procedure that was overlooked.

\section{RESULTS}

Questionnaires for ninety seven patients were successfully completed. There was no prior notification received by the referral hospital for any of the 97 patients referred and received at MTRH. Patients of all ages were referred. However, the majority, $45(46 \%)$ were in the age group of $26-40$ years. Forty three patients $(44 \%)$ were referred within 24 hours by the referring hospital.

Figure 1

Duration of patient stay in the referring facilities before transfer

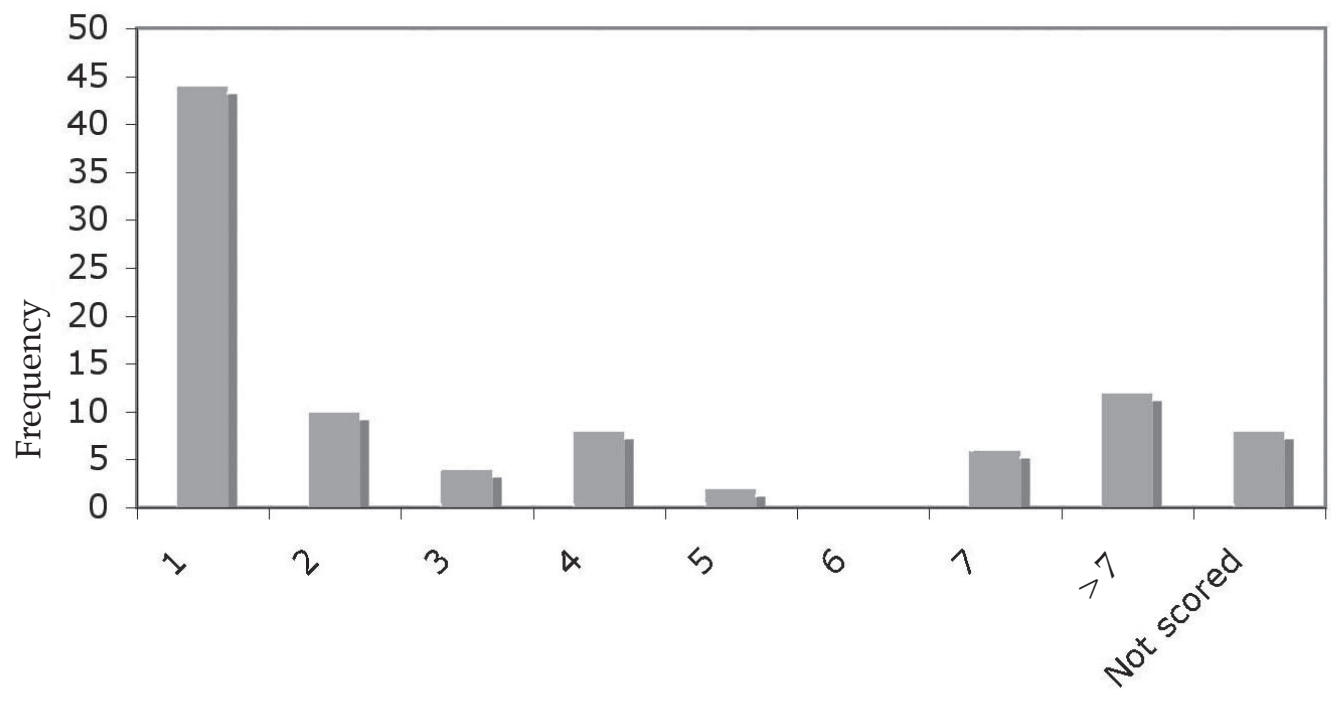

Stay in days 
Figure 2

Age distribution of patients referred to MTRH for emergency/critical care

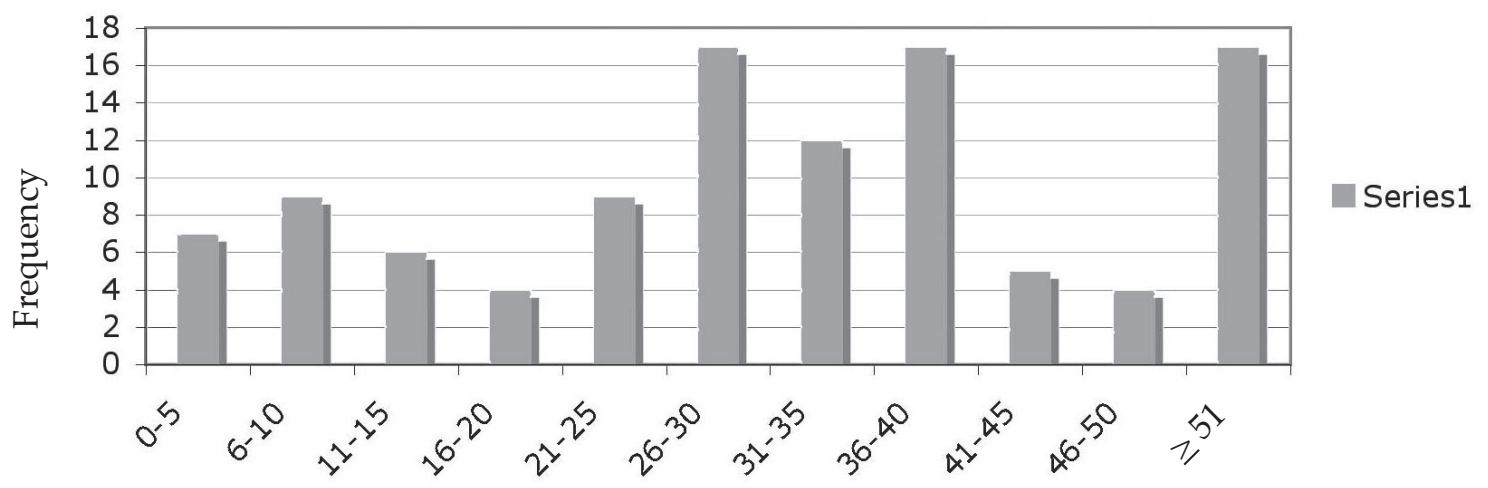

Age distribution

Figure 3

Frequency of the leading five disease conditions referred to MTRH for critical/emergency care

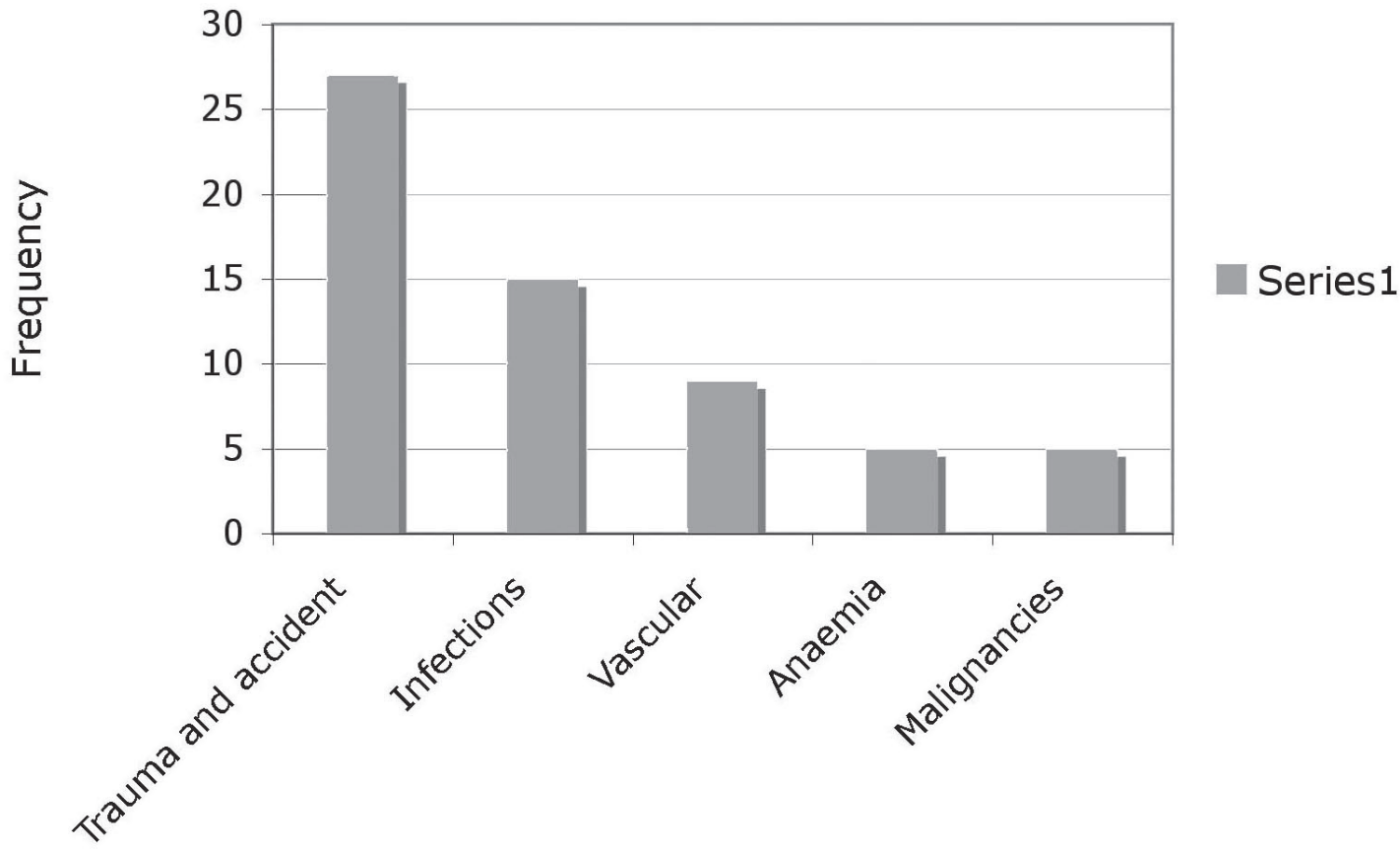

Disease condition

Fifty five $(56 \%)$ of the patients were brought in by ambulance. Others by private vehicles and public transport. Of the 97 patients, $25(26 \%)$ were conscious, $56(58 \%)$ had abnormal vital signs and $27(28 \%)$ were dehydrated requiring resuscitation fluid therapy. Trauma and accidents, vascular disorders, infections, anaemia and malignancies were the leading in order of frequency.

Appropriate escort (a nurse) was provided for $58(60 \%)$. Documentation was provided in $82(85 \%)$ of the patients. Monitoring en-route was done in 23 (24\%). Extra measures to provide warmth where required was undertaken in $59(62 \%)$ patients.

Respiratory support was required in 50 patients. Of these, seven (14\%) were provided with airway while oxygen was available for $16(32 \%)$ of them while in transit. Intravenous fluids were required in $89(94 \%)$ of the patients. Fluids were provided in $30(34 \%)$ of the patients. Venous access though required in all patients being referred was available in only $72 \%$. 
Nasogastricintubation was required in $27(28 \%)$, eight $(30 \%)$ of these patients were provided with nasogastric tube that was functional at the time of evaluation in the emergency department. Urethral catheterisation was required in $56(59 \%)$ of the patients. Thirteen $(23 \%)$ of them had catheter placed. Nine $(50 \%)$ of the patients with long bone fractures were splinted and $3 \%$ of those who required cervical spine stabilisation before confirmation of the status of the same had a collar.

Indications for specific procedures that were not carried out were recognised in $25 \%$ by the escorts. In 7\%, the procedures were in place but were discontinued once transfer was decided on. In $62 \%$ of the responses from the escorts, understaffing and lack of resources were cited as being responsible for non-application of the procedures.

\section{DISCUSSION}

Inequalities in the distribution of health services will forever dictate that patients are transferred from one hospital to another in search of better services. The underlying goal being the attainment of optimal health care and well being of the patient $(6,7)$. This however is achievable where there is no hiatus in the continuum of care during the transfer process (5).

The economic recession of the $80 \mathrm{~s}$ and $90 \mathrm{~s}$ adversely affected the health sector leading to decline in expenditure on health. The under-funding translated into performance below the optimum. There is unequal distribution of specialised services that has persisted and remained centralised despite the expansion in population and increase in disease burden.

Inadequate funding of the health sector stood out as a major constraint in the application of principles of patient transfer by hospitals in Western Kenya. This has affected staffing levels and provision of other necessary resources for efficient health care delivery including critical care transport.

Beyond the resources, inadequate knowledge and poor attitudes did contribute to the poor execution of patient transfer. Even within developed economies, innovation has been relied on to tilt the scales in favour of optimal health care delivery. Custom made facilities and equipment are expensive and it is unlikely that there will be adequate funding in the near future to satisfy the Kenyan needs. Knowledge and positive attitude drive the innovative spirit in the desired direction to solve perceived and real problems. In only $25 \%$ of the responses, were the escorts aware of the indications for specific procedures that were not carried out, while in $7 \%$ the procedures were discontinued once transfer was decided on. Splints for long bone fractures and cervical collars do not necessarily have to be factory made to serve the purpose. Villagers for example take patients to hospital with pieces of wood effectively acting as splints but can only be faulted for failure to consider the effect on circulation because they are applied without respect for this aspect of physiology. If splinting can be done at the village level, it is expected that hospitals would perform better.

Majority of patients referred to MTRH $(75 \%)$ were in the $\leq 50$ years age bracket with the majority falling in the economically productive group of $26-50$ years. This group was afflicted mostly by the trauma and infections. These two are a significant burden our health care system has to deal with though degenerative conditions in the elderly and congenital anomalies in the very young pause significant challenges. Acquired disorders and a significant proportion of congenital anomalies are amenable to treatment with excellent outcomes and therefore justify the extra effort needed to correctly apply patient transfer principles.

Before moving a patient to another hospital, he/ she should be in as stable a state as is reasonable $(1,2)$. A balance may need to be struck between treating the patient where he/ she is almost in adverse situation and the risk of transporting to better facilities. Telephonic advice in this situation will play a crucial role but this service is hardly used. Treatment started during stabilisation and support for vital functions instituted should be continued enroute (2).

The goals of monitoring are: to detect any changes in status of a patient and, therefore, be in a position to institute effective intervention, and allow for effective specialised care and support demanded by the situation.

Monitoring en-route is therefore important not only to the escort but also to the receiving personnel at the referral institution, who will be well placed to mount an immediate and appropriate response guided by the parameters of monitoring en-route. Vital signs, input and output chart monitoring will most of the time be adequate during transportation. They do not require risky procedures or use of complicated and expensive equipment considering the space, comfort and facilities available aboard the mode of transport in use. It also allows close contact of the escort and the patient, a situation that is vital for acquisition of important collateral information.

The escort of a given patient should be appropriately matched. Adverse physiological effects occur during transportation of patients and the escort ought to be one who can mount a required emergency response or make appropriate decision (6). The receiving hospital must also be in a state of preparedness to receive a critically ill patient. The state of preparedness is triggered by prior information relayed by the referring institution. Any 
documents and specimens relating to the patient ought to accompany the patient for use by the receiving institution to cut the costs in performing same tests again and ensuring that intervention is not delayed.

It is gratifying to note that prompt decisions were made to refer patients as a significant number $(44 \%)$ were referred within 24 hours. The modes of transport in some situations where ambulance was not available frustrated these well intentioned decisions. Public transport and private vehicles are hardly appropriate for transporting critically ill patients and those who require emergency services. A well funded and managed transport service in a region can effectively serve several hospitals if each individual hospital cannot be facilitated to run its own service.

In conclussion, it is evident that application of patient transfer principles fell short of what would be considered appropriate even in resource poor environments. Among other requirements, relevant knowledge and positive attitude of health workers are needed to circumvent the barrier of inadequate funding to contribute to needed improvements in patient transportation in the short term period. Improved funding of the public health sector and development of inter-hospital transfer guidelines will promote consistent high quality patient care in the region while allowing variation to meet specific local needs.

\section{ACKNOWLEDGEMENTS}

We sincerely thank Moi University for the financial support that enabled this research to be done. We also thank the Institutional Research and Ethics Committee (IREC) for valuable guidance and approving the research, hospital administration for allowing the research to be done at the hospital and all those who have contributed immensely to the success of this work. We sincerely thank Wekola Catherine Nyongesa for excellent secretarial services

\section{REFERENCES}

1. Tan, T. K. Inter-hospital and intra-hospital transfer of critically ill patients. Singapore Med. J. 1997; 38: 244-248.

2. Meller, R. M. Patient Evacuation and Dispersal. In. Medicine for Disasters. Edited by Baskett et al. Butterworth and Co. Publishers, 1998, London.

3. Stephens, C., Beehan, S. and Hillman, K. Preparation of the critically ill for transfer in MetropolitanSydney. Aust. Crit. Care. 1995; 8: 14 -18.

4. Graeme, J. D. Outcome of critically ill patients undergoing inter-hospital transfer. Med.J. Aust. 2001; 174: $122-125$.

5. Hadley, G. P. and Mars, M. Improving neonatal transport in the third world: technology or teaching? South Afr. J. Surg. 200 I: 39: 122-124.

6. Guide for interfacility patient transfer. National Highway Traffic Safety Administration. April 2006.

7. From, R. E. Jr and Dellinger, R. P. Transport of critically ill patients. Intensive Care Med. 1992; 7: 223-233. 